\title{
FIRST AID FOR MENTAL HEALTH IN GERMANY
}

\section{ERSTE HILFE FÜR PSYCHISCHE GESUNDHEIT IN DEUTSCHLAND}

\author{
Michael Deuschle \& Tabea Sarah Send \\ Zentralinstitut für Seelische Gesundheit, Universität Heidelberg, Fakultät für Medizin Mannheim, Mannheim, Germany
}

\section{SUMMARY}

Early recognition of mental disorders and psychological crisis is essential for successful therapy and good outcome of affected persons. Relatives and affected persons need health knowledge in order to identify early symptoms and psychological crisis and to guide patients to the health care system. Mental Health First Aid is a lay-based program of health education, which is evidence-based and wide-spread in English-speaking countries. In a 12-hour "Standard Course" lay persons learn basic knowledge of mental disorders and of the health care system in order to be able to access persons with beginning mental disorders or in crisis, to provide First Aid and to give advice about professional help. Currently, Mental Health First Aid is introduced in Switzerland and Germany.

Key words: health knowledge - lay person - First Aid - early recognition - MHFA - Mental Health First Aid

\begin{abstract}
ZUSAMMENFASSUNG
Die Früherkennung psychischer Störungen und psychischer Krisen ist wesentlich für eine erfolgreiche Therapie und eine günstige Prognose der Betroffenen. Es bedarf allerdings Gesundheitswissen bei Betroffenen und Angehörigen um Frühsymptome und Krisen zu erkennen und um die Patienten dem professionellen Hilfesystem zuzuführen. Mental Health First Aid ist ein laien-basiertes Programm der Gesundheitsbildung, das evidenz-basiert und im englischen Sprachraum weit verbreitet ist. In einem 12-stündigen „Basiskurs" lernen Laien Grundkenntnisse psychischer Störungen und des Hilfesystems kennen um betroffene Angehörige anzusprechen, um Erste Hilfe geben zu können und um die Betroffenen an professionelle Hilfe zu vermitteln. Das Programm Mental Health First Aid wird derzeit in der Schweiz und in Deutschland eingeführt.
\end{abstract}

Schlüsselwörter: Gesundheitswissen - Laien - Erste Hilfe Früherkennung - MHFA - Mental Health First Aid

\section{Bedeutung von Gesundheitswissen in der Allgemeinbevölkerung}

Die meisten psychischen Erkrankungen treten erstmals im frühen Jugend- und jungen Erwachsenenalter auf, nehmen häufig einen schweren, langandauernden Verlauf mit wiederkehrendem oder anhaltendem Behandlungsbedarf (Beesdo-Baum et al. 2015, Lambert et al. 2013) und sind mit früher Beeinträchtigung (Mendelson \& Eaton 2018) und vorzeitiger Mortalität (Reininghaus et al. 2015) verbunden. Deshalb entsteht durch psychische Erkrankungen eine große Krankheitslast, nicht nur für Betroffene und Angehörige sondern auch für andere Personen im Umfeld von Menschen mit psychischen Gesundheitsproblemen (Rössler et al. 2005). Häufig erleben Angehörige von Menschen mit psychischen Störungen Hilflosigkeit, weil ihnen psychische Gesundheitskompetenz fehlt, wie z.B. Wissen und Fertigkeiten, dem Angehörigen zu helfen und Rat zu geben. Die psychische Gesundheitskompetenz beinhaltet das Wissen und die Überzeugungen über psychische Erkrankungen, deren Erkennung, Management und Prävention.

\section{Was ist Erste Hilfe für psychische Gesundheit?}

Um die psychische Gesundheitskompetenz der Allgemeinbevölkerung zu verbessern, wurde im Jahr 2000 in Australien das Mental Health First Aid (MHFA) Programm, basierend auf dem Erste Hilfe Modell für körperliche Gesundheit entwickelt. Seitdem wurden über 3 Mio. Laien (d.h. Mitglieder der Allgemeinbevölkerung) in MHFA-Kursen geschult (Kitchener and Jorm 2002a). Sie werden in über 25 Ländern angeboten und vor kurzem begannen die ersten Kurse in Deutschland.

Psychische Störungen sind außerordentlich häufig. Einer von fünf Erwachsenen entwickelt pro Jahr ein Problem der psychischen Gesundheit. Durch die hohe Prävalenz psychischer Störungen haben die meisten Laien immer wieder Kontakt mit jemandem, der an einer psychischen Störung leidet (Steel et al. 2014). Oft sind es Angehörige oder Kollegen, denen die Rolle zufällt, Betroffene anzusprechen, Ersthilfe und Rat zu geben. Wegen des systematischen Trainings in körperlicher Erster Hilfe wissen die meisten Laien, was bei einem Notfall physischer Gesundheit $\mathrm{zu}$ tun ist. Hingegen ist das Wissen über Ersthilfe bei psychischen Störungen gering. MHFA-Kurs wurde im Jahr 2000 von Betty Kitchener und Tony Jorm entwickelt, um Laien Wissen und Fertigkeiten zu erster Hilfe für psychische Gesundheit zu vermitteln, mit denen sie Menschen helfen können, die ein Problem der psychischen Gesundheit entwickeln oder sich in einer psychischen Krise befinden (Kitchener \& Jorm 2002b). Die Hilfe soll angeboten werden, bis angemessene professionelle Hilfe verfügbar oder die Krise abgeklungen ist. Im Kurs lernen die Teilnehmer die Symptome verschiedener psychischer Störungen zu erkennen, Krisen psychischer 
Gesundheit einzuschätzen und Ersthilfe anzubieten. Dazu gehört es, die betroffene Person angemessener Behandlung und anderer Unterstützung zuzuführen.

\section{Was wird im Ersthelferkurs vermittelt?}

Mental Health First Aid orientiert sich an den Zielen erster Hilfe für körperliche Erkrankungen. MHFA schützt vor Gefahren, wenn eine psychische Störung zu einer Gefährdung des Betroffenen oder anderer Personen führt. Durch erste Hilfe und geeignete Ratschläge zum Aufsuchen professioneller Hilfe führt MHFA dazu, dass es nicht zu einer Verschlechterung von Gesundheitsproblemen kommt. MHFA bietet unmittelbare Unterstützung und Hilfe für eine Person mit einem psychischen Gesundheitsproblem. Es ist wichtig zu betonen, dass MHFA-ausgebildete Ersthelfer über keine diagnostische oder therapeutische Kompetenz verfügen. Die wesentlichen Lerninhalte der MHFA-Kurse sind:

- Risikofaktoren und Frühsymptome psychischer Gesundheitsprobleme;

- Information über Depression, Angststörungen, Psychose und substanzbezogene Störungen;

- Ein Handlungsplan mit fünf Bausteinen, um Menschen in einer Krise oder mit einer beginnenden psychischen Störung zu helfen;

- Verfügbare evidenzbasierte, professionelle, Peerund Selbsthilfeunterstützung zu aktivieren.

\section{Bausteine zur Ersten Hilfe für psychische Gesundheit}

Das australische Akronym des Handlungsplans in fünf Bausteinen wird im deutschsprachigen Raum als ROGER übersetzt:

$R=$ reagieren: begegnen, bewerten, beistehen;

$O=$ offen und unvoreingenommen zuhören und kommunizieren;

$G=$ gib Unterstützung und Information;

$E=$ ermutige zu professioneller Hilfe;

$R=$ reaktiviere Ressourcen.

Damit erfüllt Mental Health First Aid verschiedene Funktionen der Gesundheitsversorgung:

- Frühes Erkennen psychischer Störungen;

- Erkennen von Krisen und Beistand in Notfallsituationen;

- Unterstützung und Anbieten evidenzbasierter Maßnahmen;

- Aktivieren von Selbsthilfemaßnahmen und des sozialen Netzes.

\section{Frühes Erkennen psychischer Störungen}

Gerade bei ersterkrankten Personen ist es bedeutsam, das Störungsbild früh zu erkennen und die betroffenen Personen einer Behandlung zuzuführen. Die Dauer unbehandelter psychischer Störungen ist assoziiert mit einem ungünstigen Verlauf der Erkrankung. Dies ist gut belegt für schizophrene Psychosen, trifft aber gleichermaßen auf Patienten mit Stimmungserkrankungen und Abhängigkeitserkrankungen zu. In einigen Ländern ist Mental Health First Aid weit verbreitet (z. B. Australien: $3 \%$ der Bevölkerung). Dadurch ist es möglich, dass ein Patient, der erstmalig an einer psychischen Störung leidet, durch einen Laienhelfer rascher in Diagnostik und Therapie kommen kann. Das ist nicht nur für das unmittelbare Leid des Betroffenen bedeutsam, sondern auch für die Prognose der Erkrankung. Praktisch immer sind es Angehörige, Freunde oder Kollegen, die zuerst bemerken, wenn sich eine psychische Störung entwickelt oder eine Krise auftritt. Im Ersthelferkurs wird vermittelt, welche Symptome auf eine psychische Erkrankung hindeuten und wie eine betroffene Person angesprochen werden kann (Jorm et al. 2005).

\section{Erkennen von Krisen und Beistand in Notfallsituationen}

Bei den meisten Erkrankungen gibt es Krisen, die unmittelbares Handeln erfordern. Dies trifft auch für psychische Störungen zu. So kann es bei Depression zu akuter Suizidalität oder bei Psychosen zu akuten schweren psychotischen Zuständen kommen. Im Ersthelferkurs lernen die Laien solche Krisen zu erkennen und adäquat darauf zu reagieren. Dadurch ist Erste Hilfe für psychische Gesundheit besonders hilfreich in Notfallsituationen.

\section{Unterstützung und Anbieten evidenzbasierter Maßnahmen und Aktivieren von Selbsthilfemaßnahmen und des sozialen Netzes}

Während des gesamten Handlungsplans wird „offenes und unvoreingenommenes Zuhören und Kommunizieren“ geübt. Dieses Vorgehen erleichtert es den Betroffenen, sich zu öffnen und Hilfe anzunehmen. Ein wichtiger Teil der Wissensvermittlung besteht darin, den Ersthelfern Information $\mathrm{zu}$ geben, welche Versorgungsmöglichkeiten es gibt. Bei der Ersthilfe wird die betroffene Person aktiv ermuntert, professionelle Hilfe anzunehmen. Bei den meisten psychischen Störungen gibt es Möglichkeiten, eigene Ressourcen zu reaktivieren. Beispielsweise kann auf früher hilfreiche Strategien zurückgegriffen werden. Fast immer ist es hilfreich, das soziale Netz der betroffenen Person zu aktivieren. Im Ersthelferkurs wird das dafür notwendige Wissen vermittelt, aber auch in Rollenspielen das aktive Handeln eingeübt.

\section{Praktische Aspekte}

Der Ersthelferkurs ist ein Kurs, der aus 4 dreistündigen Blöcken besteht. Damit ist der Kurs zeitlich für Laien gut leistbar. Der Kurs wird unterstützt von standardisiertem Lehrmaterial, das sich völlig auf evidenzbasiertes Wissen stützt. Zusätzlich wird der Kurs 
unterstützt durch ein Lehrbuch, dessen Inhalt auf den Kursinhalt abgestimmt ist. Die Instruktoren, die den Laienkurs anbieten, werden nach festgelegten Kriterien ausgewählt. In der Regel ist der Instruktor eine Fachperson, die beruflich Erfahrung mit den vermittelten Lerninhalten hat (z.B. Arzt, Psychologe, Fachpflege o.ä.). Entsprechende Personen erwerben in einem 5tägigen Kurs die Qualifikation, Laienkurse anzubieten. Es gibt eine ganze Reihe von Maßnahmen, die der Qualitätssicherung dienen: online-basierte Rückmeldungen der Kursteilnehmer, jährliches Instruktorentreffen u.a.m. Nach einem Ersthelferkurs besteht die Möglichkeit, in einer kurzen Online-Prüfung als Ersthelfer akkreditiert zu werden.

\section{Welche Kurse gibt es?}

Der „Basiskurs“ ist der 12-stündige Ersthelferkurs für „Erwachsene helfen Erwachsenen“. In vielen Ländern werden zusätzliche Kurse angeboten: der ,YouthMHFA-Kurs“ ist ein 14-stündiger Kurs, in dem Erwachsene lernen, Jugendlichen zu helfen. Es gibt einen Kurs „MHFA for the elderly“, in dem Erwachsene lernen, älteren Menschen mit psychischen Störungen zu helfen (Svensson and Hansson 2017). Zusätzlich gibt es einen Kurs „Teen MHFA“, der in verkürzter Form (3,5 Stunden) geeignet ist, im Schulunterricht angeboten $\mathrm{zu}$ werden (Hart et al. 2016). In diesem Kurs lernen Teenager anderen Teenagern mit psychischen Problemen zu helfen. Diese Kurse werden üblicherweise eingeführt, wenn das Zentrum mehrere Jahre Erfahrung mit dem „Basiskurs“ gesammelt hat.

\section{Organisation von Mental Health First Aid}

Ausgehend von Australien ist MHFA vor allem in englischsprachigen Ländern weit verbreitet. Es gibt ca. 3 Mio. Ersthelfer weltweit. Die Haupthürde der weltweiten Verbreitung von MHFA sind Sprachbarrieren. Derzeit wird MHFA in der Schweiz (unter dem Namen ensa) und in Deutschland eingeführt. Üblicherweise vergibt Mental Health First Aid International einem nationalen Zentrum die Lizenz, „Instruktoren“ auszubilden. Diese Instruktoren bieten dann flächendeckend Ersthelferkurse an. Durch dieses „Train-theTraniner-System“ kann sich MHFA rasch verbreiten.

\section{Mögliche Rolle von MHFA}

Im Kontext der hohen Bedeutung der Dauer unbehandelter Psychose für die Prognose des Patienten, sprechen sich verschiedene Fachgesellschaften für Prävention und Früherkennung aus. Die WHO (World Health Organization 2001) nimmt an, dass nicht nur bei Psychose, sondern auch bei Stimmungserkrankungen und Alkoholabusus Früherkennung und Frühintervention kosteneffektive Maßnahmen sind. Dies zielt insbesondere auf jüngere Menschen, da die genannten Erkrankungen häufig eine Erstmanifestation in Adoleszenz und jungem Erwachsenenalter zeigen.
Angesichts des demographischen Wandels und der rapiden Veränderungen in der Arbeitswelt kommt dem Erhalt der psychischen Gesundheit eine zentrale Rolle zu. Die Wirksamkeit präventiver Interventionen bei psychischen Erkrankungen ist wissenschaftlich belegt. Sie können bspw. die Neuerkrankungsraten bei Depression deutlich reduzieren (Mihalopoulos et al. 2011). Zwar gibt es, vor allem in psychiatrischen Universitätskliniken, seit einigen Jahren Früherkennungsambulanzen, insbesondere für psychotische Störungen. Da jedoch gerade bei Erstmanifestation einer psychischen Störung häufig kein Krankheitsgefühl und keine Therapiemotivation vorliegt, ist es wichtig, dass Angehörige oder Freunde des Betroffenen Symptome erkennen können und den Betroffenen einer Behandlung zuführen. Bei Betroffenen und Angehörigen sind Stigma und fehlendes Gesundheitswissen wesentliche Gründe für verzögertes Aufsuchen von spezialisierten Frühinterventionsdiensten (Cabassa et al. 2018). An dieser Stelle schließt Mental Health First Aid eine Lücke der Behandlungskette.

\section{Wissenschaftliche Evidenz für MHFA}

Es gibt über MHFA ca. 100 wissenschaftliche Publikationen. Randomisierte kontrollierte Studien bei Mitgliedern der Allgemeinbevölkerung haben positive Effekte von MHFA u.a. auf ein erhöhtes Zutrauen Erste Hilfe für psychische Gesundheit zu leisten (Jorm et al. 2010) und erhöhtes Wissen über psychische Gesundheit sowie reduzierte stigmatisierende Einstellungen (Lam et al. 2010) gezeigt. Die Wirksamkeit von MHFA wurde bisher in zahlreichen Studien, Studienpopulationen und Ländern untersucht (Morgan et al. 2018). Erste Hinweise, dass MHFA auch für die Ersthelfer selbst günstige Effekte auf die psychische Gesundheit haben kann, bedürfen wissenschaftlicher Bestätigung. Ebenso gibt es in Deutschland momentan noch keine Evidenzbasierung zur Wirksamkeit von MHFA in der Allgemeinbevölkerung.

\section{Stand der Einführung in Deutschland}

Seit Januar 2019 ist das Zentralinstitut für Seelische Gesundheit in Mannheim zertifiziertes Zentrum für Mental Health First Aid. Mit Hilfe der Unterstützung der Dietmar Hopp und der Beisheim Stiftung werden derzeit Trainer ausgebildet, die Infrastruktur für die Durchführung der Instruktorenausbildung aufgebaut und die Trainingsmaterialien vorbereitet. Mit einem Start des Programms in Deutschland wird im Frühjahr 2020 gerechnet.

\section{Acknowledgements: None.}

Conflict of interest: None to declare.

\section{Contribution of individual authors:}

Equivalent contributions of both authors. 


\section{Literatur}

1. Beesdo-Baum K, Knappe S, Asselmann E, Zimmermann $P$, Brückl T, Höfler M, Behrendt S, Lieb $R \&$ Wittchen HU: The "Early Developmental Stages of Psychopathology (EDSP) Study": A 20-Year Review of Methods and Findings. SocPsychiatry Psychiatr Epidemiol 2015; 50:851-66

2. Cabassa LJ, Piscitelli S, Haselden M, Lee RJ,Essock SM \& Dixon LB: Understanding Pathways to Care of Individuals Entering a Specialized Early Intervention Service for First-Episode Psychosis. Psychiatr Serv 2018; 69:648-56

3. Hart LM, Mason RJ, Kelly CM, Cvetkovski $S$ \& Jorm AF: "Teen Mental Health First Aid": A Description of the Program and an Initial Evaluation. Int J Ment Health Syst 2016; 10:3

4. Jorm AF, Kitchener BA \& Mugford SK: Experiences in Applying Skills Learned in a Mental Health First Aid Training Course: A Qualitative Study of Participants' Stories. BMC Psychiatry 2005; 5:43

5. Jorm AF, Kitchener BA, Sawyer $M G$, Scales $H$ \& Cvetkovski S: Mental Health First Aid Training for High School Teachers: A Cluster Randomized Trial. BMC Psychiatry 2010; 10:51

6. Kitchener BA \& Jorm AF: Mental Health First Aid Training for the Public: Evaluation of Effects on Knowledge, Attitudes and Helping Behavior. BMC Psychiatry 2002, 2:10

7. Lam AY, Jorm AF \& Wong DF: Mental Health First Aid Training for the Chinese Community in Melbourne, Australia: Effects on Knowledge about and Attitudes toward People with Mental Illness. Int J Ment Health Syst 2010; 4:18
8. Lambert M, Bock T, Naber D, Löwe B, Schulte-Markwort M, Schäfer I, Gumz A, et al.: Mental Health of Children, Adolescents and Young Adults - Part 1: Prevalence, Illness Persistence, Adversities, Service Use, Treatment Delay and Consequences'. Fortschr Neurol Psychiatr 2013; 81:614-27

9. Mendelson $T$ \& Eaton $W W$ : Recent Advances in the Prevention of Mental Disorders. Soc Psychiatry Psychiatr Epidemiol 2018; 53:325-39

10. Mihalopoulos C, Vos T, Pirkis $J$ \& Carter R: The Economic Analysis of Prevention in Mental Health Programs. Annu Rev Clin Psychol 2011; 7:169-201

11. Morgan AJ, Ross A \& Reavley NJ: Systematic Review and Meta-Analysis of Mental Health First Aid Training: Effects on Knowledge, Stigma, and Helping Behaviour. PLoS One 2018; 13:e0197102

12. Reininghaus U, Dutta R, Dazzan P, Doody GA, Fearon P, Lappin J, Heslin M, et al.: Mortality in Schizophrenia and Other Psychoses: A 10-Year Follow-up of the SOP FirstEpisode Cohort. Schizophr Bull 2015; 41:664-73

13. Rössler W, Salize HJ, van Os J \& Riecher-Rössler A: Size of Burden of Schizophrenia and Psychotic Disorders. Neuropsychopharmacol 2005; 15:399-409

14. Steel Z, Marnane C, Iranpour C, Chey T, Jackson JW, Patel V \& SiloveD: The Global Prevalence of Common Mental Disorders: A Systematic Review and MetaAnalysis 1980-2013. Int J Epidemiol 2014; 43:476-93

15. Svensson B \& Hansson L: Mental Health First Aid for the Elderly: A Pilot Study of a Training Program Adapted for Helping Elderly People. Aging Ment Health 2017; 21:595-601

16. World Health Organization: Prevention and Promotion in Promotion in Mental Health. Geneva, Switzerland, 2001

\section{Correspondence:}

Prof. Michael Deuschle, MD

Zentralinstitut für Seelische Gesundheit

Klinik für Psychiatrie und Psychotherapie

J5, 68159 Mannheim, Germany

E-mail: michael.deuschle@zi-mannheim.de 